\title{
Der Zugang öffentlicher und wirtschaftlicher Interessen zum Deutschen Bundestag
}

\author{
Florian Spohr
}

Angenommen: 28. Januar 2021 / Online publiziert: 17. Februar 2021

(C) Der/die Autor(en) 2021

Zusammenfassung Dieser Beitrag untersucht den informellen und den formalisieren Zugang von öffentlichen und wirtschaftlichen Interessen zum Deutschen Bundestag im Jahr 2015. Der informelle Zugang wird über die Vergabe von Hausausweise für den Bundestag erhoben, der formalisierte Zugang über Einladungen zu öffentlichen Anhörungen der Bundestagsausschüsse. Der Beitrag geht dabei von den Annahmen des Ressourcenabhängigkeitsansatzes aus, dass Informationen und Unterstützung access goods für den Bundestag sind, welche für den Zugang zu Hausausweisen und Anhörungen unterschiedlich förderlich sind und von organisierten Interessen in unterschiedlichem Maße angeboten werden können. Die Analyse kommt zu zwei zentralen Ergebnissen: Zum einen unterscheiden sich die Zugangsmuster der unterschiedlichen Interessen. Während wirtschaftliche Interessen häufiger Hausausweise besitzen, werden öffentliche Interessen überproportional oft in öffentliche Anhörungen eingeladen. Vertreter wirtschaftlicher Interessen können spezifische Expertise und Informationen über ökonomische Bedürfnisse anbieten, die ihnen häufiger Zugang über Hausausweise eröffnen. Public Interest Groups, Gewerkschaften und Wohlfahrtsverbände hingegen werden überproportional oft in öffentliche Anhörungen eingeladen, da sie politische Unterstützung für Abgeordnete bereitstellen können. Zum anderen kommt die Analyse zu dem Ergebnis, dass Lobbying eine legislative Unterstützung von organisierten Interessen für verbündete Abgeordnete darstellt. Den unterschiedlichen Interessen kommen hierbei verschieden Rollen in Anhörungen zu. Während Regierungsfraktionen öffentliche Interessen einladen, um Regierungsinitiativen zu unterstützen, sollen wirtschaftlichen Interessen Änderungsbedarf an Gesetzesentwürfen der Regierung aufzeigen.

Dr. F. Spohr $(\bowtie)$

Fakultät für Sozialwissenschaft, Ruhr-Universität Bochum, Bochum, Deutschland

E-Mail: florian.spohr@rub.de 


\section{Access of public and business interests to the German Bundestag}

Abstract This article analyses the informal and the formal access of public and business interests to the German Bundestag in the year 2015. Informal access is surveyed by the door passes handed out by the Bundestag administration and the parliamentary party groups, formal access by the invitations to the public hearings of the Bundestag's committees. Building on resource dependence theory, this contribution assumes that information and support are access goods to the Bundestag which are of different importance for informal and formal access and unequally distributed between the different interests. The analysis yields the following results: On the one hand, access patterns differ among the interests. While business interests possess more door passes, public interests are invited disproportionately frequent to public hearings. Evidently, representatives of specific business interests offer specific expertise and information about economic needs that are of prevalent importance for the informal access, while groups advocating public interests can primarily lend support to parliamentarians in public hearings. On the other hand, the analysis indicates that lobbying is a legislative subsidy of organized interests to their allies in parliament. In this connection, parliamentary party groups assign different roles to the diverse interests in public hearings. While they invite public interests to support government initiatives, business interests are expected to point out needs of change to legislation.

\section{Einleitung}

Als im internationalen Vergleich besonders einflussreiches Parlament mit weitreichenden Möglichkeiten zur Gesetzesformulierung (Siefken und Schüttemeyer 2013, S. 162) ist der Deutsche Bundestag ein attraktives Ziel für organisierte Interessen. Um dort politische Entscheidungen in ihrem Sinne beeinflussen zu können, müssen sie allerdings Zugang zu diesem erlangen. Denn Zugang zu politischen Institutionen stellt eine notwendige Bedingung für Einflussnahme dar (Eising 2007, S. 387) und ist dadurch charakterisiert, dass er nicht nur von Organisationen gesucht, sondern zusätzlich durch Gatekeeper gewährt wird:

We propose to define access as present when a group has entered a political arena (parliament, administration, or media) passing a threshold controlled by relevant gatekeepers (politicians, civil servants, or journalists). This implies that for access to be present, interest groups need to seek it, and relevant gatekeepers need to allow it. (Binderkrantz und Pedersen 2017, S. 307)

Organisierte Interessen können auf formalisiertem und informellem Weg Zugang zu politischen Arenen bekommen (Berkhout 2013, S. 238; Binderkrantz und Pedersen 2017). Der formalisierte Zugang zum Bundestag ist über dessen Geschäftsordnung (GOBT) mit den öffentlichen Anhörungen der Bundestagsausschüsse institutionalisiert und recht gut erforscht (Weßels 1987; Müller-Rommel 1988; von Beyme 1998; Dhungel und Linhart 2014; von Winter 2014; Eising und Spohr 2017; Spohr 2018; Cross et al. 2021). Der informelle Zugang zum Bundestag kann hingegen 
schwerer identifiziert werden. Weßels (1987, S. 286) definiert informelle Kontakte der Abgeordneten als nicht institutionalisiert, aber institutionenbezogenen, also auf Vertreter*innen einer Organisation oder Profession ausgerichtet. Dass über diese „relativ wenig bekannt“ ist (Weßels 1987, S. 297-298), trifft auch drei Jahrzehnte später noch zu und liegt nicht zuletzt an der aktuell diskutierten geringen Regulierung und Dokumentation des Lobbyismus im Bundestag. Die meisten Studien erheben daher das Kontaktverhalten von Abgeordneten über Befragungen ebendieser (etwa Best et al. 2011; Gerstenhauer 2014; Weßels 2014; Wonka 2017; Wonka und Haunss 2020).

Im November des Jahres 2015 jedoch verpflichtete das Oberverwaltungsgericht Berlin-Brandenburg (nach einer Klage von abgeordnetenwatch.de, unterstützt von einer Eilklage des Berliner Tagesspiegel) die Bundestagsverwaltung dazu, offenzulegen, welche Verbände, Organisationen und Unternehmen Hausausweise für den Bundestag besitzen (Reyher 2015; Cianciara und Stasiak 2019, S. 180-181). Zuvor waren weder Verwaltung noch Fraktionen dazu verpflichtet, darüber zu informieren, an wen sie diese Ausweise, die für ein Kalenderjahr zum Betreten des Bundestagsgeländes berechtigen, ausgegeben haben (Wissenschaftliche Dienste Deutscher Bundestag 2012). Diese Daten eröffnen die Gelegenheit, den informellen Zugang zum Bundestag zu erfassen.

Diese Möglichkeit nutzend, untersucht dieser Beitrag den informellen und den formalisieren Zugang von öffentlichen und wirtschaftlichen Interessen zum Deutschen Bundestag im Jahr 2015 über die Einladungen von Sachverständigen zu öffentlichen Anhörungen und über die Vergabe von Hausausweisen durch die Bundestagsverwaltung und die Fraktionen. Dabei werden zwei Forschungsfragen zur parlamentarischen Interessenvermittlung in Deutschland verfolgt: Welche Interessen bekommen Zugang über Hausausweise und welche zu Anhörungen? Und besteht ein Zusammenhang zwischen dem Besitz von Hausausweisen der Fraktionen und der Wahrscheinlickeit einer Einladung in eine öffentliche Anhörung sowie der inhaltlichen Positionierung in dieser?

Die Analyse kommt zu zwei zentralen Ergebnissen: Erstens zeigt sie Unterschiede im Zugang von öffentlichen und wirtschaftlichen Interessen zum Bundestag auf, die auf unterschiedliche Ressourcen von wirtschaftlichen und öffentlichen Interessen zurückgeführt werden können. Arbeitgeber- und Wirtschaftsverbände sowie Unternehmen finden häufiger Zugang über Hausausweise; mutmaßlich aufgrund der spezifischen Expertise und Informationen über ökonomische Bedürfnisse, die sie anbieten können. Public Interest Groups, Gewerkschaften und Wohlfahrtsverbände hingegen werden überproportional oft in öffentliche Anhörungen eingeladen, da sie politische Unterstützung für Abgeordnete bereitstellen können.

Das zweite zentrale Ergebnis dieses Beitrags ist, dass Lobbyismus eine legislative Unterstützung von organisierten Interessen für verbündete Abgeordnete darstellt (Hall und Deardorff 2006). Organisationen mit Hausausweisen von Regierungsfraktionen haben eine signifikant höhere Chance auf eine Einladung in öffentliche Anhörungen und - mit Ausnahme wirtschaftlicher Interessen - unterstützen dort die Initiativen der Regierung. Fraktionen verschaffen offensichtlich ihnen politisch nahestehenden Interessen Zugang. 
Im folgenden Kapitel werden aus dem Ressourcenabhängigkeitsansatz Annahmen über die Bedeutung unterschiedlicher access goods für den Zugang zu Hausausweisen und Anhörungen abgeleitet. Abschnitt 3 formuliert ergänzende Hypothesen entlang der Annahme der Legislative-Subsidy-Theorie, dass Lobbyismus eine gegenseitige Unterstützung von Interessenvertreter*innen und Abgeordneten darstellt. Die empirische Analyse im vierten Kapitel prüft die Hypothesen. Das Fazit fasst die zentralen Ergebnisse zusammen und interpretiert diese.

\section{Parlamentarische access goods öffentlicher und wirtschaftlicher Interessen}

Der Ressourcenabhängigkeitsansatz konzipiert Zugang zu politischen Arenen als Produkt einer Tauschbeziehung. Interessenvertreter*innen stellen von politischen Akteuren nachgefragte Ressourcen wie Informationen, Expertise und Unterstützung bereit und erhalten im Gegenzug von diesen Legitimation, relevante policy-bezogene oder strategisch wichtige Informationen und die Aussicht auf Einflussnahme (Bouwen 2004a; Hall und Deardorff 2006; Berkhout 2013; Pedersen et al. 2014; Binderkrantz et al. 2015; Binderkrantz und Pedersen 2017). Zugang zu politischen Arenen ermöglicht Interessengruppen nicht nur, entlang der Einflusslogik, Entscheidungen in ihrem Sinne zu beeinflussen, sondern ebenfalls, entlang der Mitgliedschaftslogik, ihre Organisation für potenzielle Mitglieder attraktiver als die Konkurrenz erscheinen zu lassen (Berkhout 2013, S. 238).

Die von politischen Akteuren nachgefragten Ressourcen können unterschieden werden in für eine effektive Politikformulierung benötigte Insider-Ressourcen und in für die Außenwirkung der Politik relevante Outsider-Ressourcen (Kriesi et al. 2007; Pedersen et al. 2014; Binderkrantz et al. 2015; Flöthe und Rasmussen 2019). Die Literatur zu Interessengruppen geht davon aus, dass, entlang der fundamentalen Unterscheidung von Olson (1992), konzentrierte, ökonomische und diffuse, öffentliche Interessen diese in unterschiedlichem Maße anbieten können:

Wirtschaftliche Interessen mit konzentriertem Nutzen für ihre Mitglieder besitzen einen Vorteil im inside lobbying, da sie über spezifische Informationen verfügen, die vom Gesetzgeber nachgefragt werden (Offe und Wiesenthal 1980, S. 86; Flöthe und Rasmussen 2019, S. 828), etwa über die Effektivität und die Auswirkungen von Policies (Burstein und Hirsh 2007). Zudem besitzen Verbände, die in die Implementation von Policies eingebunden sind, nicht nur aus der Praxis gewonnene Sachkenntnis, sondern auch implizite Vetomöglichkeiten gegen eine effektive Durchführung von Policies (Spohr 2018, S. 316). Neben Expertise sind für Abgeordnete Informationen über aggregierte ökonomische Bedürfnisse wichtig (Bouwen 2004a). Da ökonomische Prosperität zur politischen Stabilität wesentlich beiträgt (Lindblom 1977), wird keine Regierung oder Fraktion eine einheitliche Ablehnung wirtschaftlicher Interessen riskieren (Weßels 1987, S. 288).

Outsider-Ressourcen können Interessengruppen anbieten, wenn sie öffentliche Unterstützung generieren können oder ihre Anliegen Nachrichtenwert besitzen (Pedersen et al. 2014; Binderkrantz et al. 2015). Der Neo-Korporatismus betont, dass Organisationen, denen es gelingt, einen Großteil ihrer potenziellen Mitglieder zu or- 
ganisieren oder mobilisieren, mit Zugang zu politischen Institutionen belohnt werden (Berkhout 2013, S. 231). Interessen, die eine breite Öffentlichkeit betreffen, haben einen positiven Einfluss auf die öffentliche Meinung, spezifische Interessen hingegen einen negativen (Page et al. 1987, S. 37; Flöthe und Rasmussen 2019, S. 828).

Während die Exekutive vorranging an für die Politikformulierung relevante Informationen interessiert ist, fragt die Legislative sowohl Insider- als auch OutsiderRessourcen nach (Bouwen 2004b; Burstein und Hirsh 2007; Binderkrantz et al. 2015). Vor allem Arbeitsparlamente wie der Deutsche Bundestag mit ausgeprägten Kompetenzen in der Gesetzesformulierung ${ }^{1}$ benötigen externen Sachverstand, auch um dem Informationsvorsprung der ressourcenstarken Ministerialbürokratien entgegenzuwirken (Siefken und Schüttemeyer 2013, S. 171). Zwar werden Bundestagsabgeordnete durch ihre wissenschaftlichen Mitarbeiter*innen und die Fraktionsreferent*innen der Arbeitsgruppen unterstützt und können den wissenschaftlichen Dienst des Bundestages zu Rate ziehen; dennoch sind die diesbezüglichen Ressourcen des Bundestages begrenzt (Strässer und Meerkamp 2015, S. 219).

Zudem sind Abgeordnete stärker als Ministerialbürokrat*innen an politischer Unterstützung, Legitimation und medialer Präsenz (Bouwen 2004b; Pedersen et al. 2014) sowie an Informationen über die Auswirkungen von Policies auf ihre Wiederwahlchancen interessiert (Burstein und Hirsh 2007, S. 177). Wurde politische Repräsentation von Wähler*innen früher entlang der Wahlkreise als territorial gesehen, konstituieren sich in modernen Demokratien Interessen zunehmend funktional über geographische Grenzen hinweg. Thomassen und Andeweg (2004, S. 49-51) wenden daher das Konzept der politischen Repräsentation auf die Bemühungen von Abgeordneten an, Vorteile für bestimmte Gruppen ihrer Wählerschaft zu schaffen. Und die Aggregation und Repräsentation funktional definierter Interessen ist die originäre Domäne von Organisationen.

Hier stellt sich nun die Frage, welche Ressourcen organisierte Interessen im Tausch für Hausausweise anbieten können, und welche für die Einladung in Anhörungen förderlich sind. Anhörungen können von Ausschüssen, Unterausschüssen und Enquête-Kommissionen durchgeführt werden, wobei die Ausschussanhörungen klar dominieren (Siefken 2018, S. 174-175). Mehr als zwei Drittel von diesen werden als Teil des Gesetzgebungsprozesses durchgeführt (Siefken und Schüttemeyer 2013, S. 169). Die Anhörungen haben zunächst die Funktion einer öffentlich inszenierten Wissensproduktion (Sack und Fuchs 2014, S. 162). Gemäß der Selbstdarstellung des Bundestages sollen die Ausschussmitglieder durch Anhörungen ,ergänzende Informationen erhalten und in die Lage versetzen werden, ihr Abstimmungsverhalten auf der Basis möglichst vollständiger Informationen über die erwarteten Wirkungen des Gesetzes festzulegen“".2 Dieses legt die Einladung von Sachverständigen, die über Insider-Ressourcen verfügen, nahe.

Da öffentlichen Anhörungen jedoch relativ spät in der Politikformulierung stattfinden, liegt ihre Funktion weniger darin, neue Informationen in den Gesetzge-

\footnotetext{
${ }^{1}$ Zwischen dem 8. und dem 14. Deutschen Bundestag (1976-2002) wurden fast $68 \%$ der Gesetzentwürfe der Regierung durch das Parlament abgeändert (König und Bräuninger 2005, S. 15).

2 Deutscher Bundestag: Funktionen und Aufgaben der ständigen Ausschüsse; online verfügbar unter: https://www.bundestag.de/ausschuesse18/aufgaben/441892. Zugegriffen: 11. Mai 2020.
} 
bungsprozess einfließen zu lassen; dieses ist in aller Regel bereits an anderer Stelle passiert (Siefken 2018, S. 175). ${ }^{3}$ So schreibt Ismayr (2012, S. 411), dass sich die Regierungsmehrheit ,,in der Regel auch durch Kritik in öffentlichen Anhörungen allenfalls zu marginalen Änderungen bewegen“ lässt, wenn sie sich auf einen Gesetzesentwurf festgelegt hat. ${ }^{4}$ Auch wenn es in ihnen meist nur noch um Korrekturen oder Ergänzungen geht, sind öffentliche Anhörungen ,gleichwohl schon aus Gründen der Transparenz wichtige Veranstaltungen“ (Strässer und Meerkamp 2015, S. 227). Durch ihre Öffentlichkeit kommt ihnen eine Legitimationsfunktion zu. Die Anhörung von Sachverständigen dient der Untermauerung (oder der Kritik an) der politischen Machbarkeit, der sozialen Akzeptanz und der demokratischen Legitimität von Sachentscheidungen. Anhörungen bedienen dabei sowohl die Legitimitätserfordernisse der Abgeordneten gegenüber den Wähler*innen, als auch von Interessengruppen gegenüber der Politik und den eigenen Mitgliedern (Siefken und Schüttemeyer 2013, S. 171; Sack und Fuchs 2014, S. 162-164; Strässer und Meerkamp 2015; Spohr 2018). Werden Anhörungen vorrangig als öffentliche Arenen verstanden, gewinnen Outsider-Ressourcen an Relevanz (Binderkrantz et al. 2015, S. 99). Somit ist insgesamt anzunehmen, dass in öffentliche Anhörungen sowohl Sachverständige mit Insider- als auch mit Outsider-Ressourcen eingeladen werden.

Während diese Überlegungen auf ein ausgewogenes Verhältnis von wirtschaftlichen und öffentlichen Interessen in Anhörungen schließen lassen, legen drei Gründe nahe, dass vor allem wirtschaftliche Interessen Zugang über Hausausweise finden. Erstens sind gemäß neo-pluralistischer und korporatistischer Ansätze konzentrierte Interessen organisationsfähiger als diffuse Interessen (Olson 1992). Sie verfügen über mehr Ressourcen wie Geld und Mitarbeiter*innen (Binderkrantz et al. 2015), die notwendig sind, um informelle Kontakte aufzubauen und Zugang zu forcieren. Zweitens eröffnen Hausausweise im Gegensatz zu Anhörungseinladungen keinen Zugang zu einer öffentlichen Arena, in der Outsider-Ressourcen besonders relevant sind. Sie ermöglichen es jedoch schon zu einem früheren Zeitpunkt in der parlamentarischen Politikformulierung, spezifische Expertise in den Gesetzgebungsprozess einfließen zu lassen, welche gemäß der oben angeführten Annahme des Ressourcenabhängigkeitsansatzes wirtschaftliche Interessen im größeren Maße als öffentliche Interessen anbieten können.

Mehr noch legt drittens die fehlende Öffentlichkeit bei Hausausweisen nahe, dass der Austausch von Informationen über ökonomische Bedürfnisse eher informell stattfindet. Wirtschaftsverbände und Unternehmen präferieren in ihrem Lobbying quiet politics. Sie sind einflussreicher, wenn Themen weniger öffentlich sind, da sie spezifische ökonomische Interessen vertreten, die auf Kosten der Allgemeinheit gehen können (Culpepper 2010, S. 177; De Bruycker 2017, S. 607; siehe auch Kriesi et al. 2007). Und aufgrund ihrer fundamentalen Bedeutung für ökonomische Prosperität und somit auch politische Stabilität (Lindblom 1977) finden Wirtschafts-

\footnotetext{
3 Jedoch kann Anhörungen auch eine Agenda-Setting-Funktion zukommen. Gut ein Drittel der Anhörungen thematisieren allgemeine Problemlagen, aus denen gesetzgeberischer Handlungsbedarf entstehen kann (Siefken 2018, S. 178-179).

4 Quantitative Studien stellen jedoch signifikante Gesetzesänderungen als Folge öffentlicher Anhörungen fest (Eising und Spohr 2017; Cross et al. 2021).
} 
interessen auch mit der öffentlichen Meinung entgegengesetzten Positionen Gehör: „(O)rganized interests may be able to offer resources and benefits to policy makers, which outweigh the benefits of making policy that is congruent with the views of the majority of citizens" (Rasmussen et al. 2018, S. 158). Diese für die Leistungsfähigkeit des Parlaments funktional notwendigen Diskussionen fänden ,hinter verschlossenen Türen“ statt (Sarcinelli 2011, S. 272), hinter denen die Politik vor allem die Interessen der Wirtschaft berücksichtige (Crouch 2008, S. 10).

Aus den theoretischen Überlegungen zum Ressourcentausch im Parlament und den Spezifika der beiden Austauscharten Anhörungen und Hausausweise ergeben sich zusammengefasst die Annahmen, dass vorranging die über Insider-Ressourcen verfügenden wirtschaftlichen Interessen Zugang über Hausausweise finden, während die Outsider-Ressourcen öffentlicher Interessen in Anhörungen gefragter sind:

Hypothese 1a Der Anteil von Vertreter*innen wirtschaftlicher Interessen liegt bei den ausgestellten Hausausweisen höher als bei den öffentlichen Anhörungen.

Hypothese 1b Der Anteil von Vertreter*innen öffentlicher Interessen liegt bei den öffentlichen Anhörungen höher als bei den ausgestellten Hausausweisen.

\section{Lobbying als legislative Unterstützung von Abgeordneten}

Die Tauschlogik impliziert, dass beide Tauschpartner ihre Ressourcen selektiv und strategisch einsetzen. Interessenvertreter*innen werden sich überlegen, wem sie ihre Informationen und Unterstützung bereitstellen, und Abgeordnete, wem sie ihre rare Zeit widmen und Einflussmöglichkeiten bieten. Die Interessengruppenforschung hat hierbei die erstaunliche Beobachtung gemacht, dass Interessengruppen zwar gelegentlich versuchen, Abgeordnete zu überzeugen, die unentschlossen sind oder ihren Interessen entgegenstehende Standpunkte vertreten, aber in der Regel diejenigen lobbyieren, die ihre Position bereits teilen (Austen-Smith und Wright 1994, S. 25-26). Diesen kontraintuitiven Befund erklären Hall und Deardorff in ihrer LegislativeSubsidy-Theorie, in der sie Lobbyismus als , a matching grant of policy information, political intelligence, and legislative labor to the enterprises of strategically selected legislators“ (Hall und Deardorff 2006, S. 69) konzipieren. Lobbyist*innen stellen ihre Informationen hauptsächlich den politischen Akteuren zur Verfügung, deren Positionen mit ihren eigenen kongruent sind, um diese im parlamentarischen Willensbildungsprozess und demokratischen Wettbewerb zu stärken. Lobbyismus stellt so eine legislative Unterstützung in Form von Expertise und Sacharbeit für verbündete Abgeordnete dar (siehe auch Hojnacki und Kimball 1998). Marshall (2010) stützt diese Argumentation, indem er zeigt, dass Interessengruppen im Europäischen Parlament ihre politischen Verbündeten mit hochwertigeren Informationen versorgen als ihre politischen Gegner. Abgeordnete wiederum sind an dieser policy-bezogenen Zuarbeit von gleichgesinnten Lobbyist*innen interessiert (Hall und Deardorff 2006, S. 75). So haben die Mitglieder des Bundestages vorranging Verbindungen $\mathrm{zu}$ den Interessengruppen, deren technisches und politisches Wissen sie zur Stärkung ihrer eigenen Position und zur Erreichung ihrer politischen Ziele nut- 
zen können (Wonka 2017; Wonka und Haunss 2020). Demnach wäre zu erwarten, dass die Abgeordneten denjenigen Interessenorganisationen Zugang zum Bundestag gewähren, die ihnen inhaltlich nahestehen.

Bis zur Verschärfung der Zugangsbedingungen im Jahr $2016^{5}$ gab es unterschiedliche Gatekeeper, die Hausausausweise für den Bundestag ausstellen konnten. Auf dem regulären Weg wurden Hausausweise von der Bundestagsverwaltung an Verbände vergeben - unter der Voraussetzung, dass diese in der sogenannten Lobbyliste, der öffentliche Verbändeliste gemäß Anlage 2 GOBT, eingetragen sind (Wissenschaftliche Dienste Deutscher Bundestag 2012, S. 4). Die Bewilligung lag im Ermessen der Verwaltung. Bis zum Jahr 2016 konnten aber auch die Bundestagsfraktionen die Ausstellung der Hausausweise für Dritte beantragen. Um Kontakt zu Abgeordneten und Fraktionen herzustellen, hatten Lobbyist*innen , unter bestimmten Voraussetzungen die Möglichkeit, auf Antrag einen Hausausweis zu erhalten“. Hierfür mussten

Interessenvertreter (sog. Lobbyisten) (...) mit einem durch eine/n Parlamentarische/n Geschäftsführer/in (Sicherheitsbeauftragte/r) einer Fraktion gezeichneten Antrag nachweisen können, dass sie die Gebäude des Deutschen Bundestages nicht zuletzt im Interesse des Parlaments häufig aufsuchen müssen.

(Wissenschaftliche Dienste Deutscher Bundestag 2012, S. 4)

Der Zugang über die Fraktionen hatte für Interessenorganisationen den Vorteil, dass sie so mehrere Hausausweise erhalten konnten. Ferner konnten so auch andere Akteure als die in der Lobbyliste eingetragenen Verbände Hausausweise erhalten, etwa Firmen, Agenturen und Kanzleien. Gemäß der Legislative-Subsidy-Theorie wäre nun zu erwarten, dass Lobbyist*innen Hausausweise bei ihnen nahestehende Fraktionen beantragen, um diese mit Informationen zu versorgen, während Fraktionen Hausausweise an Organisationen ausgeben, die ihre politischen Standpunkte teilen.

Während der Zugang für Interessenvertreter*innen über Hausausweise nicht kontingentiert ist, ist dieser zu den Anhörungen limitiert, da üblicherweise die Ausschüsse die Anzahl der angehörten Sachverständigen begrenzen. Nach $\S 70$ Abs. 2 GOBT benennen die Fraktionen diese dann gemäß ihres Stärkeverhältnisses. Auch wenn die Ausschüsse bei bestimmten Themen eine Reihe von Spitzen- und Fachverbänden nicht ignorieren können (Ismayr 2012, S. 411), stellen somit die Fraktionen bzw. deren Obleute und Berichterstatter*innen in den Ausschüssen die Gatekeeper zu den Anhörungen dar.

Hier stellt sich die Frage, nach welchen Kriterien diese die Sachverständigen auswählen. Es ist naheliegend, das Legislative-Subsidy-Argument, welches ,,policy information, political intelligence and legislative labor" (Hall und Deardorff 2006, S. 75) umfasst, auch auf die argumentative Unterstützung von Interessenverteter*innen in öffentlichen Anhörungen anzuwenden. Dieses steht auch im Einklang

\footnotetext{
5 Anfang 2016 hat der Bundestag die Regelungen über den Zutritt von Interessenvertreter*innen zum Bundestag restriktiver ausgestaltet. Die Ausstellung der Hausausweise ist nun über die Ausweisstelle zentralisiert und auf Verbände beschränkt, die in der Lobbyliste eingetragen sind (Cianciara und Stasiak 2019, S. 180-181). Andere Akteure wie Unternehmen, Public Affairs Agenturen und Kanzleien können keine Hausausweise mehr beantragen.
} 
mit empirischen Beobachtungen, nach denen die Fraktionen häufig eine Anhörung solcher Sachverständigen wünschen, ,von denen sie (bis zu einem gewissen Grad) eine argumentative Unterstützung für ihre eigene Position erwarten“ (Ismayr 2012, S. 411). Sie rechtfertigen dann ihre politischen Positionen über die Argumente der eingeladenen Akteure (Siefken und Schüttemeyer 2013; Strässer und Meerkamp 2015). Hall und Deardorff (2006, S. 81) betonen ebenfalls, dass Abgeordnete zur Verfolgung ihrer eigenen Ziele nicht nur Informationen, sondern auch die Fähigkeit der Interessenverteter*innen zur Repräsentation nutzen. Hieraus folgt:

Hypothese 2a Interessenvertreter*innen mit Hausausweisen von Fraktionen haben eine höhere Wahrscheinlichkeit in eine öffentliche Anhörung eingeladen zu werden als Interessenvertreter*innen mit Hausausweisen der Verwaltung.

Ferner ist zu beachten, dass insbesondere bei polarisierenden Gesetzesvorhaben Anhörungen weniger die Funktion einer Kompromissfindung zwischen den verschiedenen Fraktionen und den unterschiedlichen Interessen haben als der Rechtfertigung gegenüber der Wählerschaft. Die eingeladenen Interessenvertreter*innen dienen dann ,,nur der Außendarstellung der Position, ohne dass festgefahrene Positionen der Fraktionen noch aufgebrochen werden könnten“ (Strässer und Meerkamp 2015, S. 227). Öffentliche Anhörungen fungierten dann als „Forum der (Re-)Produktion von Legitimität“, in welchem bereits getroffene Entscheidungen ,nachholend als sachangemessen öffentlich dargestellt" werden (Sack und Fuchs 2014, S. 163). Trifft dieses zu, werden Regierungsfraktionen gemäß der Lobbying-Subsidy-Theorie verbündete Akteure einladen, die ihren Standpunkt stützen. Ein Zusammenhang zwischen der Vergabe von Hausausweisen und der Positionierung von Sachverständigen in den öffentlichen Anhörungen wäre wahrscheinlich:

Hypothese 2b Interessenvertreter*innen in öffentlichen Anhörungen positionieren sich positiver zu Gesetzesvorhaben, wenn sie Hausausweise der diese initiierenden oder unterstützenden Fraktionen besitzen.

\section{Empirische Analyse}

Die empirische Analyse überprüft die in den vorherigen Abschnitten formulierten Hypothesen. Die Einladungen von Interessengruppen und anderen Sachverständigen in die öffentlichen Anhörungen der ständigen Ausschüsse des Deutschen Bundestages wurden vom Forschungsprojekt Arenen der politischen Interessenvermittlung in Deutschland (Eising und Spohr 2017; Spohr 2018; Cross et al. 2021) erhoben und kodiert. Für die Analyse der Hausausweisvergabe wurden die von abgeordnetenwatch.de veröffentlichten Datensätze ${ }^{6}$ integriert und kodiert. Um die Vergleichbarkeit der Daten zu gewährleisten wurden die 135 öffentlichen Anhörungen der Bundestagsausschüsse im Veröffentlichungsjahr der Hausausweisdaten 2015 analysiert. Im 18. Bundestag, der im Oktober 2013 gewählt wurde, bildeten die Unionsparteien

\footnotetext{
6 Die Daten sind online verfügbar unter: https://www.abgeordnetenwatch.de/blog/lobbyliste. Zugriffen: 22. April 2019.
} 
CDU und CSU mit der SPD die Regierung, während Die Linke und Bündnis 90/Die Grünen die Opposition stellten. Die FDP zog erstmalig seit Gründung der Bundesrepublik nicht in den Bundestag ein.

Die Akteure, die über Hausausweise oder Anhörungen Zugang zum Bundestag hatten, werden in sieben Kategorien klassifiziert. Die ersten drei davon vertreten wirtschaftliche Interessen und können primär für die Gesetzgebung relevante Insider-Ressourcen anbieten. Erstens vertreten Arbeitgeber- und Wirtschaftsverbände aggregierte sektorale Interessen gegenüber Gewerkschaften und dem Staat und sind zudem in die Implementation einiger Policies eingebunden. Im Zuge der Erosion korporatistischer Interessenvermittlung nimmt jedoch direktes Lobbying von, zweitens, Unternehmen zu (Speth 2015, S. 158). Diese verfügen über spezifische Informationen, da sie im Gegensatz zu Verbänden direkt im Markt aktiv sind (Bouwen 2004a, S. 478). Und drittens bringen vermehrt Public Affairs Agenturen und Rechtsanwaltskanzleien als Lobby-Dienstleister die Anliegen von Verbänden und Unternehmen in den politischen Prozess ein (Baruth und Schnapp 2015, S. 255; Speth 2015, S. 155-158; Opitz 2018).

Öffentliche Interessen werden von Public Interest Groups, inklusive NGOs, vertreten. Dieses sind gemeinnützige und allgemeinwohlorientierte Zusammenschlüsse von Mitgliedern ohne materielles Interesse an den Gruppenzielen (Zimmer und Speth 2009, S. 291), etwa Menschenrechtsorganisationen, Natur- und Verbraucherschutzgruppen. Obwohl einige Public Interest Groups und NGOs über spezifische Expertise verfügen - so sieht Schiffers (2019, S. 17) den „Bezug zu Fachexpertise als politischem Instrument und als Legitimationsargument im eigenen Selbstverständnis“ als Besonderheit von NGOs - können Public Interest Groups als Vertreterinnen bestimmter Ideale oder öffentlicher Güter vorrangig Outsider-Ressourcen anbieten; vor allem wenn ihre Anliegen populär und in den Medien präsent sind (De Bruycker 2017; Flöthe und Rasmussen 2019; Rasmussen et al. 2018).

Sowohl über Insider- als auch über Outsider-Ressourcen verfügen zum einen Gewerkschaften ${ }^{7}$ und Wohlfahrtsverbände. Als korporative Akteure, die im Neokorporatismus ähnliche Funktionen ausüben, besitzen sie ein interessenpolitisches Repräsentationsmonopol in sozialpolitisch wichtigen Domänen und werden als Leistungsanbieter in die Implementation eingebunden (Backhaus-Maul 2000). Und zum anderen Berufsverbände, da diese sowohl die wirtschaftlichen als auch sozialen und gesellschaftlichen Interessen einer Berufsgruppe oder Branche in der Öffentlichkeit vertreten. Gewerkschaften und Berufsverbände können als Vertreter sektoraler Interessen für den Gesetzgebungsprozess relevanten Sachverstand anbieten (Offe und Wiesenthal 1980, S. 86; Pedersen et al. 2014, S. 206). Flöthe und Rasmussen (2019) zeigen aber auch, dass die Forderungen dieser beiden Akteurstypen einen ähnlich starken öffentlichen Rückhalt finden, wie die von Public Interest Groups (und einen

\footnotetext{
7 Die Zuordnung von Gewerkschaften zu wirtschaftlichen oder öffentlichen Interessen ist nicht eindeutig. Während etwa De Bruycker (2017) sie als wirtschaftliche Interessen sieht, zählen Schiffers und Körner (2019) sie zu öffentlichen Interessen. Zwar vertreten Gewerkschaften die wirtschaftlichen Interessen ihrer Mitglieder, aber aufgrund der negativen Koalitionsfreiheit in Deutschland fordern sie mit besseren Arbeitsbedingungen Güter, welche nicht nur diesen zur Verfügung stehen. Zudem vertreten Gewerkschaften aufgrund der Erwartungshaltung ihrer Mitglieder und aus Working-Class-Solidarität zunehmend auch die Interessen prekär beschäftigter „Outsider“ im dualen deutschen Arbeitsmarkt (Marx und Starke 2017).
} 
stärkeren als die von Wirtschaftsverbänden und Unternehmen). „The fact that some types of interest groups represent narrower public constituencies does not disqualify them from acting in line with public preferences altogether" (Flöthe und Rasmussen 2019, S. 842).

Die letzte Kategorie sind Sonstige Akteure. In diese fallen Interessengruppen, die nicht in eine der oben genannten Kategorien fallen, etwa religiöse Gruppen, Zusammenschlüsse von Trägern öffentlicher Einrichtungen, oder Gruppen, die Freizeitinteressen wie Sport oder Kultur vertreten, sowie die übrigen nichtverbandlichen Akteure, wie wissenschaftliche Sachverständige, Stiftungen, Think-Tanks sowie Vertreter*innen von Behörden und Institutionen.

Zur Überprüfung der ersten beiden Hypothesen zum Zusammenhang von Akteurstypen und Zugangswegen vergleicht Tab. 1 die Einladungen in Anhörungen und die Vergabe von Hausausweisen. In der Spalte „Gesamt“ sind alle 1462 externen Akteure, die über Hausausweise oder öffentliche Anhörungen im Jahr 2015 Zugang zum Bundestag hatten, nach Akteurstypen dargestellt. ${ }^{8}$ Den größten Anteil der Akteure stellen die 340 Wirtschaftsverbände, gefolgt von 190 Public Interest Groups und 167 Unternehmen. In der Zeile „Anhörungen“ sind die 801 Akteure, die in öffentliche Anhörungen eingeladen wurden, dargestellt. „Hausausweise“ umfasst alle 836 externen Akteure, die im Jahr 2015 mindestens einen Hausausweis besaßen, der entweder von der Bundestagsverwaltung oder von den Fraktionen ausgestellt wurde.

Die Ergebnisse in Tab. 1 stützen zunächst Hypothese 1a, dass wirtschaftliche Interessen eher über Hausausweise als über Anhörungen Zugang zum Bundestag finden. Arbeitgeber- und Wirtschaftsverbände sowie Unternehmen weisen bei den Hausausweisen einen etwa dreimal so hohen Anteil der Akteure (35,89\% bzw. $16,27 \%$ ) wie in den Anhörungen (12,73\% bzw. 5,37\%) auf. 300 der insgesamt 340 Arbeitgeber- und Wirtschaftsverbände verfügten im Jahr 2015 über mindestens einen Hausausweis, aber nur 102 von ihnen wurden in eine öffentliche Ausschussanhörung eingeladen. Und von den insgesamt 167 Unternehmen besaßen 136 einen Hausausweis, aber nur 43 nahmen an einer Anhörung teil. Lediglich Kanzleien und Agenturen stellen mit 5,74\% einen exakt gleichen Anteil sowohl in den Anhörungen als auch bei den Hausausweisen.

Werden jedoch nur die von den Fraktionen vergebenen Hausausweise betrachtet, ergibt sich ein anderes Bild. Die Fraktionen vergaben prozentual in etwa so viele Hausausweise an Arbeitgeber- und Wirtschaftsverbände (13,58\%), wie auch in Anhörungen eingeladen wurden. Bei Kanzleien und Agenturen hingegen ist der Anteil nun fast doppelt $(10,97 \%)$, bei Unternehmen sogar mehr als sechsmal so groß $(34,73 \%)$. Dass Unternehmen, Kanzleien und Agenturen fast ausschließlich ihre Hausausweise von den Fraktionen bekommen haben liegt zunächst darin begründet, dass die Hausausweisvergabe durch die Verwaltung in der Regel auf Verbände beschränkt ist, und der Weg über die Fraktionen so ihr einziger Zugang zu Hausausweisen war. ${ }^{9}$ Dass dennoch auch in absoluten Zahlen etwa dreimal so viele Unternehmen Zugang über Hausausweise der Fraktionen bekommen haben (133)

\footnotetext{
8 Zudem haben die Fraktionen Hausausweise an ihre eigenen Parteien und den parteinahen Stiftungen ausgegeben. Da dieses keine externen Akteure darstellen, werden diese in der Analyse nicht berücksichtigt.

9 Eine Übersicht über die Vergabepraxis der unterschiedlichen Fraktionen bietet Tab. 4 im Anhang.
} 


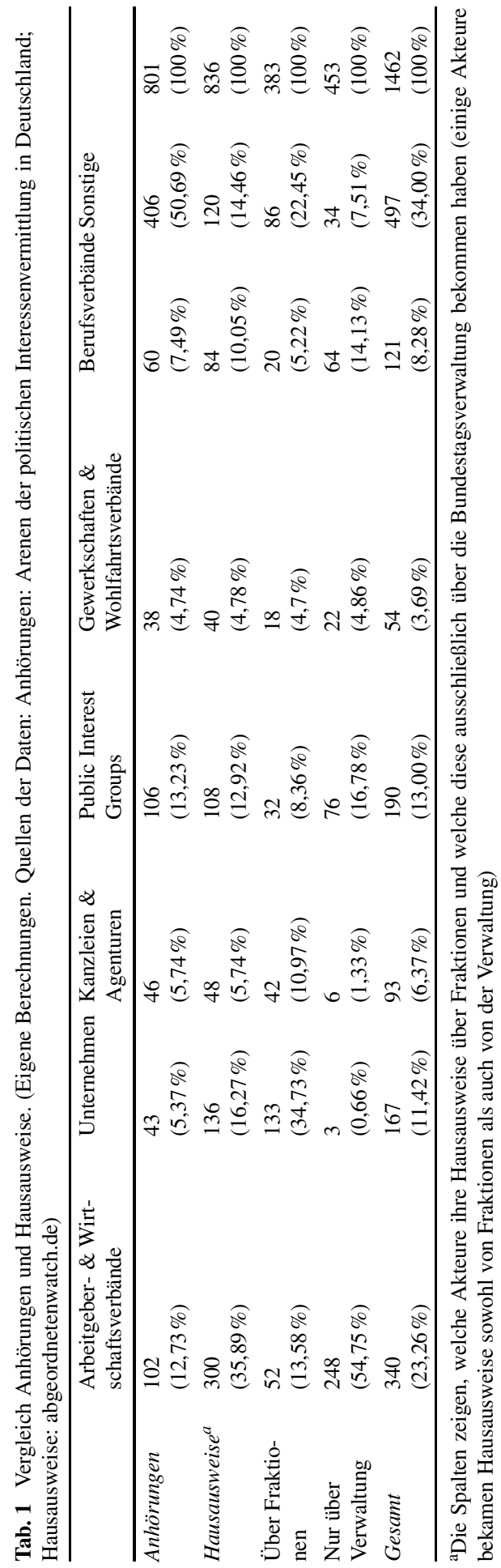


wie in Anhörungen eingeladen wurden (43) zeigt aber auch, dass Fraktionen beim Austausch mit Unternehmen offensichtlich quiet politics bevorzugen.

Die Daten in Tab. 1 widerlegen zwar die in Hypothese 1b formulierte Annahme, dass der Anteil öffentlicher Interessen in Anhörungen größer ist als bei den ausgegebenen Hausausweisen, denn Public Interest Groups stellen bei beiden Zugangsarten einen ähnlich großen Anteil an der Gesamtpopulation (13,23\% in Anhörungen und $12,92 \%$ der Hausausweise). Jedoch vergeben die Fraktionen lediglich 8,36\% ihrer Hausausweise an Public Interest Groups. Offensichtlich verschaffen Fraktionen Public Interest Groups eher Zugang zu Anhörungen, was mit deren Outsider-Ressourcen erklärt werden kann. Bei Gewerkschaften und Wohlfahrtsverbänden, die gemäß den oben formulierten Überlegungen sowohl über Insider- als auch über Outsider-Ressourcen verfügen, ist der Anteil in Anhörungen (4,74\%) fast identisch mit dem bei Hausausweisen $(4,78 \%)$, welche auch in etwa gleichem Maße von Verwaltung und Fraktionen ausgegeben wurden. Berufsverbände fanden etwas mehr informell $(10,05 \%)$ als formalisiert $(7,49 \%)$ Zugang. Der große Anteil der sonstigen Akteure in den Anhörungen liegt vor allem an individuellen, meist universitären, Experten, die mit 32,21\% fast ein Drittel aller Akteure stellen.

Als nächstes wird die Annahme der Legislative-Subsidy-Theorie überprüft, dass Lobbyismus eine Unterstützung von Organisationen für Abgeordneten mit gleichen Präferenzen darstellt. Trifft dieses zu, wäre zu erwarten, dass Fraktionen ihnen nahestehenden Lobbyist*innen nicht nur Hausausweise ausstellen, damit diese ihnen inhaltlich zuarbeiten können, sondern auch in öffentliche Anhörungen einladen, um dort ihre Positionen zu untermauern. Dementsprechend überprüft eine Regressionsanalyse, ob gemäß Hypothese 2a Interessenvertreter*innen mit Hausausweisen der Fraktionen eine höhere Wahrscheinlichkeit haben, in eine öffentliche Anhörung eingeladen zu werden, als Organisationen mit Hausausweisen der Verwaltung. Die Gesamtpopulation der Analyse besteht aus den 836 Akteuren, die 2015 mindestens einen (von der Bundestagsverwaltung oder den Fraktionen ausgestellten) Hausausweis besaßen. Die dichotom codierte abhängige Variable ist die Präsenz in mindestens einer Anhörung in dem Jahr. Die fünf unabhängigen Variablen sind die Hausausweise, die die parlamentarischen Geschäftsführer*innen der Bundestagsfraktionen und der CSU-Landesgruppe ausgestellt haben. Diese Variablen sind nummerisch codiert, da die Anzahl der ausgestellten Hausausweise von 0-15 (Hausausweise der SPD für den DGB) reicht. Zudem werden als Kontrollvariablen die Akteurstypen in die Analyse aufgenommen, da hier Verzerrungen zu erwarten sind. Tab. 4 im Anhang zeigt, dass die Fraktionen ihre Hausausweise in unterschiedlichem Maße an die verschiedenen Interessen vergeben haben.

Die Analyse in Tab. 2 beginnt mit den Kontrollvariablen. Modell 1 misst die Wahrscheinlichkeit der verschiedenen Akteurstypen in eine Anhörung eingeladen zu werden. Hierfür werden zu den sechs Akteurstypen jeweils Dummy-Variablen gebildet. Die Referenzkategorie bilden die sonstigen Akteure; gegenüber diesen wird das Chancenverhältnis der anderen Akteure in einer Anhörung präsent zu sein, geschätzt. Da in diese Kategorie weitere Interessengruppen und andere individuelle und korporative Akteure fallen, stellt sie einen fundierten Durchschnitt als Interpretationsfolie für etwaige Abweichungen dar. Das Modell zeigt für Gewerkschaften und Wohlfahrtsverbände eine um $376 \%$ erhöhte Wahrscheinlichkeit, dass diese auch 
Tab. 2 Binomiale logistische Regression mit Präsenz in Anhörungen als abhängige Variable

\begin{tabular}{|c|c|c|c|c|c|c|}
\hline & Modell 1 & & Modell 2 & & Modell 3 & \\
\hline & $\begin{array}{l}\text { Odds } \\
\text { Ratio }\end{array}$ & Std. Err & $\begin{array}{l}\text { Odds } \\
\text { Ratio }\end{array}$ & Std. Err & $\begin{array}{l}\text { Odds } \\
\text { Ratio }\end{array}$ & Std. Err \\
\hline Hausausweise CDU & - & - & $1,16325 * *$ & 0,063621 & $1,348483 * * *$ & 0,0891123 \\
\hline Hausausweise CSU & - & - & 1,237067 & 0,2194005 & $1,775855 * * *$ & 0,2882717 \\
\hline Hausausweise SPD & - & - & $1,581439 * * *$ & 0,178016 & $1,848757 * * *$ & 0,3206685 \\
\hline $\begin{array}{l}\text { Hausausweise } \\
\text { Grüne }\end{array}$ & - & - & 1,877399 & 0,6161255 & 2,041977 & 0,7493097 \\
\hline $\begin{array}{l}\text { Hausausweise } \\
\text { Linke }\end{array}$ & - & - & 0,6091788 & 0,4412949 & 0,385696 & 0,3172373 \\
\hline $\begin{array}{l}\text { Arbeitgeber- \& } \\
\text { Wirtschaftsverbän- } \\
\text { de }\end{array}$ & 0,8264271 & 0,2118025 & - & - & 1,671661 & 0,5092964 \\
\hline Unternehmen & $0,3070078 * *$ & 0,1135307 & - & - & $0,2923378 * *$ & 0,1194633 \\
\hline $\begin{array}{l}\text { Kanzleien \& Agen- } \\
\text { turen }\end{array}$ & $0,0674984 * *$ & 0,0697107 & - & - & $0,0408014 *$ & 0,0561411 \\
\hline $\begin{array}{l}\text { Public Interest } \\
\text { Groups }\end{array}$ & 0,9064039 & 0,2850835 & - & - & 1,98358 & 0,7062881 \\
\hline $\begin{array}{l}\text { Gewerkschaften \& } \\
\text { Wohlfahrtsverbän- } \\
\text { de }\end{array}$ & $4,758621 * * *$ & 1,840048 & - & - & $8,806909 * * *$ & 3,751467 \\
\hline Berufsverbände & 1,216092 & 0,3949908 & - & - & $2,465137^{*}$ & 0,9105486 \\
\hline Constant & $0,3152174 * * *$ & 0,067129 & $0,2062099 * * *$ & 0,0213744 & $0,1294914 * * *$ & 0,0364592 \\
\hline$N$ & 836 & - & 836 & - & 836 & - \\
\hline LR chi2 & 61,75 & - & 34,61 & - & 117,85 & - \\
\hline Prob $>$ chi 2 & 0,0000 & - & 0,0000 & - & 0,0000 & - \\
\hline Pseudo R2 & 0,0720 & - & 0,0403 & - & 0,1374 & - \\
\hline Log likelihood & $-398,04997$ & - & $-411,61808$ & - & $-369,99763$ & - \\
\hline
\end{tabular}

$* p<0,05 ; * * p<0,01 ; * * * p<0,001$

in Anhörungen präsent sind - was angesichts der korporatistischen Konzentration auf wenige Organisationen nicht überrascht. Bei Unternehmen mit Hausausweis ist die Wahrscheinlichkeit, auch in eine Anhörung eingeladen zu werden, um $69 \%$ geringer als bei der Referenzkategorie der sonstigen Akteure, bei Kanzleien und Agenturen um 93\%. Die Wahrscheinlichkeit der restlichen drei Akteurstypen ist ebenfalls geringer, jedoch sind die Ergebnisse nicht signifikant.

Modell 2 untersucht, wie sich die von den Fraktionen ausgestellten Hausausweise auf die Wahrscheinlichkeit einer Einladung in eine Anhörung auswirken. Hier sind die Effekte der von CDU und SPD ausgestellten Hausausweise signifikant. Mit jedem zusätzlichen Hausauweis der CDU steigt die Wahrscheinlichkeit um $16 \%$ in eine Anhörung eingeladen zu werden; und mit jedem der SPD um 58\%. Die geringere Wahrscheinlichkeit der CDU-Hausausweise erklärt sich mit der Vielzahl, die sie ausgegeben hat; 609 der 875 von den parlamentarischen Geschäftsführer*innen ausgestellten Hausausweise kamen von der CDU (s. Tab. 4 im Anhang). Nur bei den Linken ist die (nicht signifikante) Wahrscheinlichkeit, mit einem Hausausweis in eine Anhörung eingeladen zu werden, geringer als bei der CDU. 
In dem kompletten Modell 3 wird zudem nach Akteurstypen kontrolliert, um Verzerrungen aufgrund dieser confounder zu eliminieren. Hier ergeben sich auch für die von der CSU ausgestellten Hausausweisen signifikante Ergebnisse. Zudem werden stärkere positive Einflüsse (erhöhte Odds-Ratio) der Anzahl der Hausausweise auf die Teilnahme an Anhörungen geschätzt. Nun erhöht jeder zusätzliche CDU-Hausauweis die Wahrscheinlichkeit in eine Anhörung eingeladen zu werden um $35 \%$, jeder der CSU um 78\%, und jeder der SPD um 85\%. Jeder Ausweis der Grünen erhöht die Wahrscheinlichkeit sogar um $104 \%$, während jeder der Linken diese um $61 \%$ verringert. Die Ergebnisse zu den beiden Oppositionsparteien sind jedoch nicht signifikant (vermutlich aufgrund der geringen Zahl von 32 bzw. 15 Hausausweisen, die diese vergeben haben (vgl. Tab. 4 im Anhang) auf der einen und der relativ geringen Zahl von Sachverständigen, die sie gemäß ihres Sitzanteils einladen können, auf der anderen Seite). Mit Ausnahme der Linken stützen die Ergebnisse Hypothese 2a, dass Interessenvertreter*innen mit Hausausweisen von Fraktionen mit einer höheren Wahrscheinlichkeit in eine öffentliche Anhörung eingeladen werden als solche mit Hausausweisen der Verwaltung. Dieses indiziert, dass Fraktionen als die zentralen Gatekeeper in Anhörungen solche Sachverständige einladen, deren Positionen sie kennen.

Aber spiegelt sich dieses auch in der inhaltlichen Positionierung der eingeladenen Akteure wider? Zur Prüfung der Hypothese 2b, dass Interessenvertreter*innen sich in öffentlichen Anhörungen positiver zu Gesetzesvorhaben positionieren, wenn sie Hausausweise der diese initiierenden oder unterstützenden Fraktionen besitzen, werden die Positionen der eingeladenen Sachverständigen zu dem gesamten Gesetzesvorhaben anhand der vorab eingereichten schriftlichen Stellungnahmen bestimmt.

Die aus den Stellungnahmen hervorgehenden Positionen zu den Gesetzentwürfen und Anträgen der Regierung wurden durch geschulte Kodierer*innen manuell anhand eines Codebooks in eine Ordinalskala eingeordnet. ${ }^{10}$ Diese Skala umfasst fünf Kategorien, welche von (-2) bis (2) reichen, wobei (-2) komplette Ablehnung, $(-1)$ wesentliche Vorbehalte, (0) neutrale oder keine Positionierung, (1) Zustimmung mit Korrekturwünschen und (2) volle Zustimmung bedeutet. Insgesamt kennzeichnet also ein positiver Wert eine zustimmende und ein negativer Wert eine ablehnende Position.

In Tab. 3 gibt die Spalte „Alle Akteure“ die durchschnittliche Position aller Sachverständigen in den Anhörungen zu Gesetzesentwürfen oder Anträgen der Regierung oder ihren Fraktionen im Jahr 2015 wieder, ${ }^{11}$ die mittlere Spalte die durchschnittliche Position aller Sachverständigen mit von der Bundestagsverwaltung oder den Parteien ausgestellten Hausausweisen, und die Spalte ganz rechts die durchschnittliche Position aller Sachverständigen mit von den Regierungsfraktionen CDU/CSU und SPD ausgestellten Hausausweisen.

Die Tabelle zeigt, dass sich Public Interest Groups, Berufsverbände, Gewerkschaften und Wohlfahrtsverbände deutlich positiver zu den Anträgen und Gesetzes-

\footnotetext{
10 Ein Test der Intercoder-Reliabilität mittels Krippendorf's Alpha ergab einen Wert von 0,869, was ein akzeptables Niveau aufzeigt.

11 Da nicht alle Akteure Stellungnahmen eingereicht haben und manche Anhörungen Anträge und Gesetzesentwürfe der Opposition thematisieren, reduziert sich das $n$ auf 819 .
} 
Tab. 3 Positionen der Akteure zu Gesetzesentwürfen oder Anträgen der Regierung(sfraktionen). (Eigene Berechnungen. Quelle der Daten: Arenen der politischen Interessenvermittlung in Deutschland)

\begin{tabular}{|c|c|c|c|c|}
\hline & & $\begin{array}{l}\text { Alle } \\
\text { Akteure }\end{array}$ & $\begin{array}{l}\text { Akteure mit } \\
\text { Hausausweisen }\end{array}$ & $\begin{array}{l}\text { Akteure mit Haus- } \\
\text { ausweisen der } \\
\text { Regierungsfraktionen }\end{array}$ \\
\hline \multirow{2}{*}{$\begin{array}{l}\text { Arbeitgeber- \& } \\
\text { Wirtschaftsverbände }\end{array}$} & Position & $-0,0526316$ & $-0,0964912$ & $-0,16$ \\
\hline & Anzahl & 152 & 114 & 50 \\
\hline \multirow[t]{2}{*}{ Unternehmen } & Position & $-0,3703704$ & $-0,6$ & $-0,6$ \\
\hline & Anzahl & 27 & 10 & 10 \\
\hline \multirow{2}{*}{$\begin{array}{l}\text { Agenturen \& } \\
\text { Kanzleien }\end{array}$} & Position & $-0,1875$ & 0 & 0 \\
\hline & Anzahl & 32 & 1 & 1 \\
\hline \multirow[t]{2}{*}{ Public Interest Groups } & Position & $-0,0105263$ & 0,1621622 & 0,5454545 \\
\hline & Anzahl & 95 & 37 & 11 \\
\hline \multirow{2}{*}{$\begin{array}{l}\text { Gewerkschaften \& } \\
\text { Wohlfahrtsverbände }\end{array}$} & Position & 0,1123596 & 0,1388889 & 0,2222222 \\
\hline & Anzahl & 89 & 72 & 45 \\
\hline \multirow[t]{2}{*}{ Berufsverbände } & Position & 0,0470588 & 0,02 & 0,2105263 \\
\hline & Anzahl & 85 & 50 & 19 \\
\hline \multirow[t]{2}{*}{ Sonstige } & Position & 0,1477506 & 0,2714728 & 0,2835821 \\
\hline & Anzahl & 339 & 70 & 67 \\
\hline \multirow[t]{2}{*}{ Gesamt } & Position & 0,2709677 & 0,0536723 & 0,1231527 \\
\hline & Anzahl & 819 & 354 & 203 \\
\hline
\end{tabular}

entwürfen der Regierung positionieren, wenn sie Hausausweise besitzen. Wie in Hypothese $2 \mathrm{~b}$ erwartet fällt ihre Position sogar noch positiver aus, wenn nur die Akteure mit Hausausweisen der Regierungsparteien betrachtet werden. Die Regierungsfraktionen vergaben somit Hausausweise an organisierte Interessen, die ihre Positionen eher teilen und in den öffentlichen Anhörungen ihre Vorhaben unterstützen. Dieses gilt jedoch nicht für ökonomische Interessen. Diese positionieren sich nicht nur deutlich kritischer als die anderen Akteure; die Position von Arbeitgeberund Wirtschaftsverbänden sowie Unternehmen ist sogar noch ablehnender, wenn sie Hausausweise der Regierungsparteien besitzen. Offensichtlich laden Mitglieder der Regierungsfraktionen wirtschaftliche Interessen dann ein, wenn sie Veränderungsbedarf an den Gesetzesentwürfen der Ministerien sehen. ${ }^{12}$

\section{Fazit}

Die Analyse des Zugangs organisierter Interessen zum Deutschen Bundestag anhand der Einladungen zu öffentlichen Anhörungen der Bundestagsausschüsse und der

\footnotetext{
${ }^{12}$ Martin und Vanberg (2004) zeigen, dass Koalitionsparteien in Parlamentsausschüssen Korrekturen an Gesetzesentwürfen aus den Ressorts ihrer Koalitionspartner vornehmen. Dieses trifft hier aber nicht zu, denn sowohl Arbeitgeber- und Wirtschaftsverbände als auch Firmen mit Hausausweisen der CDU positionieren sich zu Entwürfen aus den Ressorts der Unionsparteien kritischer $(-0,333)$ als zu denen aus den sozialdemokratischen Ressorts $(-0,267)$. Ebenso positionieren sich Akteure mit Hausausweisen der SPD zu den Entwürfen der sozialdemokratischen Ressorts kritischer $(-0,4)$ als zu denen der Ressorts der Union $(-0,214)$.
} 
Vergabe von Hausausweisen durch die Bundestagsverwaltung und die Fraktionen im Jahr 2015 kommt zu zwei zentralen Ergebnissen.

Erstens zeigt sie systematische Unterschiede im Zugang von öffentlichen und wirtschaftlichen Interessen zum Bundestag auf, die auf unterschiedliche Ressourcen der verschiedenen Interessen zurückgeführt werden können. Wirtschaftsinteressen fanden mehr Zugang über Hausausweise als zu Anhörungen. Dieses kann mit ihren Insider-Ressourcen erklärt werden, denn sie besitzen nicht nur für eine effektive Politikformulierung benötigte Sachkenntnis, sondern auch Informationen über ökonomische Bedürfnisse, mit denen sie die öffentliche Meinung ausstechen können (Rasmussen et al. 2018, S. 158). Daher präferieren wirtschaftliche Interessen quiet politics (Culpepper 2010); und offensichtlich tauschen sich Abgeordnete insbesondere mit Firmen, Kanzleien und Agenturen auch eher nichtöffentlich aus.

Dass die Fraktionen jeden dritten Hausausweis an Unternehmen ausgestellt haben, kann auch damit erklärt werden, dass letztere aufgrund ihrer Finanzkraft mehr Möglichkeiten als andere Akteure haben, diesen Zugang zu forcieren. So eröffnen immer mehr große Unternehmen Hauptstadtrepräsentanzen in Berlin, um Abgeordnete direkt zu kontaktieren (Strässer und Meerkamp 2015). Letztlich spiegelt das Übergewicht für wirtschaftliche Interessen bei den Hausausweisen auch das aus der Forschung bekannte Verhältnis in der Gesamtpopulation wider, nachdem in etwa dreimal so viele wirtschaftliche wie öffentliche Interessenorganisationen (inklusive Gewerkschaften) existieren (für einen Überblick siehe Schiffers und Körner 2019).

Public Interest Groups finden so auch einen ihrem Anteil an der Gesamtpopulation der Interessengruppen äquivalenten informellen Zugang über Hausausweise, was andeutet, dass sie auch über spezifische Sachkenntnis verfügen (Schiffers 2019, S. 17). Sie werden aber vor allem von den Fraktionen überproportional oft in öffentliche Anhörungen eingeladen. Eine Erklärung hierfür ist, dass sie über mehr auf der offenen Bühne relevante Outsider-Ressourcen wie Nachrichtenwert und Unterstützung verfügen. Eine andere ist, dass die Abgeordneten offensichtlich darum bemüht sind, in den Anhörungen das aufgrund unterschiedlicher Organisationsfähigkeiten existierende Ungleichgewicht zwischen ökonomischen und anderen Interessen (Olson 1992) auszugleichen und beiden in gleichem Maße Zugang zu ermöglichen. Dieses ist im Einklang mit den Befunden von Pedersen et al. (2015) zu den Konsultationspraktiken in britischen, dänischen und niederländischen Parlamentsausschüssen, in denen geschlossener Zugang durch gezielte Einladungen die Diversität der Akteure erhöht, während bei offenem Zugang durch open calls Wirtschaftsinteressen dominieren.

Das zweite zentrale Ergebnis dieses Beitrags ist, dass Lobbying eine legislative Unterstützung von organisierten Interessen für verbündete Abgeordnete darstellt (Hall und Deardorff 2006). Fraktionen verschaffen Interessen Zugang, die ihnen inhaltlich nahestehen. Dieses zeigt sich nicht nur darin, dass die Vergabe von Hausausweisen durch die Fraktionen die bekannte Nähe von konservativen Parteien zu Arbeitgeber- und linker Parteien zu Arbeitnehmer- und öffentlichen Interessen bestätigt (Spohr 2019; vgl. Tab. 4 im Anhang), sondern auch darin, dass Organisationen mit Hausausweisen aller Fraktionen außer der Linken eine erhöhte Chance auf eine Einladung in öffentliche Anhörungen haben. Das Parteiinteresse, verbündeten Organisationen Zugang zu verschaffen, spielte somit bei der Vergabe der Hausausweise 
und der Auswahl der angehörten Organisationen offensichtlich eine zentrale Rolle. Zudem befürworten Public Interest Groups, Berufsverbände, Gewerkschaften und Wohlfahrtsverbände Initiativen der Regierungsfraktionen stärker, wenn diese ihnen Hausausweise ausgestellt haben. Fraktionen laden diese Interessen offensichtlich in Anhörungen ein, damit diese ihre Position bestätigen. Die Fähigkeit zur Legitimierung politischer Vorhaben ist somit ein wichtiges access good zu Anhörungen.

Eine bemerkenswerte Ausnahme hiervon stellen wirtschaftliche Interessen dar. Diese positionieren sich deutlich kritischer zu Anträgen und Gesetzesentwürfen der Regierung - und sogar noch ablehnender, wenn sie Hausausweise von deren Fraktionen bekommen haben. Dieses deutet darauf hin, dass den unterschiedlichen Interessen in öffentlichen Anhörungen unterschiedliche Funktionen zukommen (vgl. Sack und Fuchs 2014, S. 165). Bei bereits erfolgter Festlegung der politischen Entscheidung laden die Regierungsfraktionen überwiegend Interessen ein, welche mit ihren Outsider-Ressourcen die Gesetzesentwürfe darstellen und öffentlich legitimeren können. Bei noch offenen Entscheidungen oder Konflikten zwischen Ausschüssen und Ministerien hingegen ist externe Expertise wichtig für den Bundestag, um den Ministerien als Ursprungsort der Gesetzesentwürfe entgegenzuwirken (Siefken und Schüttemeyer 2013, S. 171). Und hierbei sind wirtschaftliche Interessen aufgrund ihren Insider-Ressourcen mehr als andere Akteure change agents, deren Kritik zu Änderungen an den Ministerialentwürfen führt. Entsprechend zeigen auch Studien, dass Kritik von wirtschaftlichen Interessen in Anhörungen signifikant zu mehr Gesetzesänderungen führt als Kritik von öffentlichen Interessen (Eising und Spohr 2017; Cross et al. 2021).

Die Ergebnisse zeigen somit weiteren Forschungsbedarf zu der Rolle parlamentarischer Interessenvertretung bei der Wissensproduktion für und Legitimation von Policies sowie im Zusammenspiel von Regierung und Parlament auf. Hierfür könnten etwa die Positionen von organisierten Interessen zu verschiedenen Aspekten der Gesetzesvorhabens differenziert werden. Zudem könnte stärker nach den Eigenschaften der diskutierten Policies kontrolliert werden, etwa dem Konfliktgrad und deren redistributive oder regulative Wirkung. Vor allem aber bedarf es der Erschließung weiterer Datenquellen zu den Kontakten von Abgeordneten zu organisierten Interessen, um das Bild zu komplettieren. Eine generell offene Frage ist, wie Unternehmen, Public Affairs Agenturen und Kanzleien, die seit der Neuregulierung der Hausausweisvergabe im Jahr 2016 keine Hausausweise mehr beantragen können, nun informellen Zugang zum Bundestag finden. Daher besteht weiterer Bedarf an Regulierungen, insbesondere an der Einführung eines Lobbyregisters, welches die Kontakte von organisierten Interessen zu Abgeordneten dokumentiert und deren finanzielle Lobbyaufwendungen offenlegt (Ebener 2020). Für die Transparenz und Input-Legitimation der parlamentarischen Gesetzgebung wäre dieses ein ebenso förderlicher Schritt wie für die politikwissenschaftliche Forschung zur Interessenvermittlung in der deutschen Volksvertretung.

Funding Open Access funding enabled and organized by Projekt DEAL.

Open Access Dieser Artikel wird unter der Creative Commons Namensnennung 4.0 International Lizenz veröffentlicht, welche die Nutzung, Vervielfältigung, Bearbeitung, Verbreitung und Wiedergabe in jeglichem Medium und Format erlaubt, sofern Sie den/die ursprünglichen Autor(en) und die Quelle ord- 
nungsgemäß nennen, einen Link zur Creative Commons Lizenz beifügen und angeben, ob Änderungen vorgenommen wurden.

Die in diesem Artikel enthaltenen Bilder und sonstiges Drittmaterial unterliegen ebenfalls der genannten Creative Commons Lizenz, sofern sich aus der Abbildungslegende nichts anderes ergibt. Sofern das betreffende Material nicht unter der genannten Creative Commons Lizenz steht und die betreffende Handlung nicht nach gesetzlichen Vorschriften erlaubt ist, ist für die oben aufgeführten Weiterverwendungen des Materials die Einwilligung des jeweiligen Rechteinhabers einzuholen.

Weitere Details zur Lizenz entnehmen Sie bitte der Lizenzinformation auf http://creativecommons.org/ licenses/by/4.0/deed.de. 


\section{Anhang}

Tab. 4 Vergabe von Hausausweisen durch die im Bundestag vertretenen Parteien. (Eigene Berechnungen. Quelle der Daten: abgeordnetenwatch.de)

\begin{tabular}{|c|c|c|c|c|c|c|c|}
\hline Akteure $^{a}$ & & $\mathrm{CDU}$ & $\mathrm{CSU}$ & SPD & Grüne & Linke & Gesamt $^{\mathrm{b}}$ \\
\hline \multirow{2}{*}{$\begin{array}{l}\text { Arbeitgeber- \& } \\
\text { Wirtschaftsver- } \\
\text { bände }\end{array}$} & Akteure & $\begin{array}{l}38 \\
(13,97 \%)\end{array}$ & $\begin{array}{l}3 \\
(7,14 \%)\end{array}$ & $\begin{array}{l}15 \\
(17,65 \%)\end{array}$ & $\begin{array}{l}2 \\
(7,69 \%)\end{array}$ & 0 & $\begin{array}{l}52 \\
(13,58 \%)\end{array}$ \\
\hline & Ausweise & $\begin{array}{l}95 \\
(15,6 \%)\end{array}$ & $\begin{array}{l}7 \\
(9,09 \%)\end{array}$ & $\begin{array}{l}18 \\
(11,84 \%)\end{array}$ & $\begin{array}{l}2 \\
(6,25 \%)\end{array}$ & 0 & $\begin{array}{l}122 \\
(13,94 \%)\end{array}$ \\
\hline \multirow{2}{*}{$\begin{array}{l}\text { Gewerkschaften } \\
\& \text { Wohlfahrts- } \\
\text { verbände }\end{array}$} & Akteure & $\begin{array}{l}11 \\
(4,04 \%)\end{array}$ & 0 & $\begin{array}{l}5 \\
(5,88 \%)\end{array}$ & $\begin{array}{l}3 \\
(11,54 \%)\end{array}$ & $\begin{array}{l}2 \\
(15,39 \%)\end{array}$ & $18(4,7 \%)$ \\
\hline & Ausweise & $\begin{array}{l}20 \\
(3,28 \%)\end{array}$ & 0 & $\begin{array}{l}22 \\
(14,47 \%)\end{array}$ & $\begin{array}{l}3 \\
(9,38 \%)\end{array}$ & $\begin{array}{l}3 \\
(20 \%)\end{array}$ & $\begin{array}{l}48 \\
(5,49 \%)\end{array}$ \\
\hline \multirow[t]{2}{*}{ Berufsverbände } & Akteure & $\begin{array}{l}14 \\
(5,15 \%)\end{array}$ & $\begin{array}{l}3 \\
(7,14 \%)\end{array}$ & $\begin{array}{l}3 \\
(3,53 \%)\end{array}$ & $\begin{array}{l}1 \\
(3,85 \%)\end{array}$ & 0 & $\begin{array}{l}20 \\
(5,22 \%)\end{array}$ \\
\hline & Ausweise & $\begin{array}{l}38 \\
(6,24 \%)\end{array}$ & $\begin{array}{l}3 \\
(3,90 \%)\end{array}$ & $\begin{array}{l}11 \\
(7,24 \%)\end{array}$ & $\begin{array}{l}1 \\
(3,13 \%)\end{array}$ & 0 & $\begin{array}{l}53 \\
(6,06 \%)\end{array}$ \\
\hline \multirow[t]{2}{*}{$\begin{array}{l}\text { Public Interest } \\
\text { Groups }\end{array}$} & Akteure & $\begin{array}{l}19 \\
(6,99 \%)\end{array}$ & $\begin{array}{l}1 \\
(2,38 \%)\end{array}$ & $\begin{array}{l}8 \\
(9,41 \%)\end{array}$ & $\begin{array}{l}5 \\
(19,23 \%)\end{array}$ & $\begin{array}{l}7 \\
(53,85 \%)\end{array}$ & $\begin{array}{l}32 \\
(8,36 \%)\end{array}$ \\
\hline & Ausweise & $\begin{array}{l}15 \\
(3,12 \%)\end{array}$ & $\begin{array}{l}1 \\
(1,30 \%)\end{array}$ & $\begin{array}{l}8 \\
(5,26 \%)\end{array}$ & $\begin{array}{l}5 \\
(15,63 \%)\end{array}$ & $\begin{array}{l}7 \\
(46,67 \%)\end{array}$ & $\begin{array}{l}40 \\
(4,57 \%)\end{array}$ \\
\hline \multirow[t]{2}{*}{ Unternehmen } & Akteure & $\begin{array}{l}104 \\
(38,24 \%)\end{array}$ & $\begin{array}{l}19 \\
(45,24 \%)\end{array}$ & $\begin{array}{l}21 \\
(24,71 \%)\end{array}$ & $\begin{array}{l}4 \\
(15,39 \%)\end{array}$ & $\begin{array}{l}1 \\
(7,69 \%)\end{array}$ & $\begin{array}{l}133 \\
(34,73 \%)\end{array}$ \\
\hline & Ausweise & $\begin{array}{l}203 \\
(33,33 \%)\end{array}$ & $\begin{array}{l}34 \\
(44,16 \%)\end{array}$ & $\begin{array}{l}26 \\
(17,10 \%)\end{array}$ & $\begin{array}{l}5 \\
(15,63 \%)\end{array}$ & $\begin{array}{l}1 \\
(6,67 \%)\end{array}$ & $\begin{array}{l}269 \\
(30,74 \%)\end{array}$ \\
\hline \multirow[t]{2}{*}{$\begin{array}{l}\text { Kanzleien \& } \\
\text { Agenturen }\end{array}$} & Akteure & $\begin{array}{l}34 \\
(12,50 \%)\end{array}$ & $\begin{array}{l}7 \\
(16,67 \%)\end{array}$ & $\begin{array}{l}3 \\
(3,53 \%)\end{array}$ & $\begin{array}{l}2 \\
(7,69 \%)\end{array}$ & 0 & $\begin{array}{l}42 \\
(10,97 \%)\end{array}$ \\
\hline & Ausweise & $\begin{array}{l}88 \\
(14,45 \%)\end{array}$ & $\begin{array}{l}17 \\
(22,08 \%)\end{array}$ & $\begin{array}{l}3 \\
(1,97 \%)\end{array}$ & $\begin{array}{l}2 \\
(6,25 \%)\end{array}$ & 0 & $\begin{array}{l}110 \\
(12,57 \%)\end{array}$ \\
\hline \multirow[t]{2}{*}{ Sonstige } & Akteure & $\begin{array}{l}52 \\
(19,12 \%)\end{array}$ & $\begin{array}{l}9 \\
(21,43 \%)\end{array}$ & $\begin{array}{l}30 \\
(35,29 \%)\end{array}$ & $\begin{array}{l}9 \\
(34,62 \%)\end{array}$ & $\begin{array}{l}3 \\
(23,08 \%)\end{array}$ & $\begin{array}{l}86 \\
(22,45 \%)\end{array}$ \\
\hline & Ausweise & $\begin{array}{l}146 \\
(23,97 \%)\end{array}$ & $\begin{array}{l}15 \\
(19,48 \%)\end{array}$ & $\begin{array}{l}64 \\
(42,10 \%)\end{array}$ & $\begin{array}{l}14 \\
(43,76 \%)\end{array}$ & $\begin{array}{l}4 \\
(26,67 \%)\end{array}$ & $\begin{array}{l}243 \\
(27,20 \%)\end{array}$ \\
\hline \multirow[t]{2}{*}{ Total } & Akteure & $\begin{array}{l}272 \\
(100 \%)\end{array}$ & $\begin{array}{l}42 \\
(100 \%)\end{array}$ & $\begin{array}{l}85 \\
(100 \%)\end{array}$ & $\begin{array}{l}26 \\
(100 \%)\end{array}$ & $\begin{array}{l}13 \\
(100 \%)\end{array}$ & $\begin{array}{l}383 \\
(100 \%)\end{array}$ \\
\hline & Ausweise & $\begin{array}{l}609 \\
(100 \%)\end{array}$ & $\begin{array}{l}77 \\
(100 \%)\end{array}$ & $\begin{array}{l}152 \\
(100 \%)\end{array}$ & $\begin{array}{l}32 \\
(100 \%)\end{array}$ & $\begin{array}{l}15 \\
(100 \%)\end{array}$ & $\begin{array}{l}875 \\
(100 \%)\end{array}$ \\
\hline
\end{tabular}

aDie Zeile „Akteure“ zählt die Anzahl der Organisationen, die Hausausweise erhalten haben. Da einige Akteure mehrere Zugangsberechtigungen erhielten, zählt „Ausweise“ die Gesamtzahl an Hausausweisen, die Fraktionen diesem Akteurstyp ausgestellt haben

${ }^{b}$ Da einige Akteure von mehreren Parteien Hausausweise bekommen haben, ist die Anzahl der gesamten Akteure niedriger als die Summe der Parteien 


\section{Literatur}

Austen-Smith, David, und John R. Wright. 1994. Counteractive lobbying. American Journal of Political Science 38:25-44.

Backhaus-Maul, Holger. 2000. Wohlfahrtsverbände als korporative Akteure. Aus Politik und Zeitgeschichte B26(27):22-30.

Baruth, Stephanie, und Kai-Uwe Schnapp. 2015. Ministerialbürokratien als Lobbyadressaten. In Lobby Work. Interessenvertretung als Politikgestaltung, Hrsg. Rudolf Speth, Anette Zimmer, 245-260. Wiesbaden: Springer VS.

Berkhout, Joost. 2013. Why interest organizations do what they do: assessing the explanatory potential of 'exchange' approaches. Interest Groups \& Advocacy 2(2):227-250.

Best, Heinrich, Michael Edinger, Daniel Gerstenhauer, und Lars Vogel. 2011. Jenaer Parlamentarierbefragung 2010. Ausgewählte Ergebnisse. Sonderforschungsbereich 2010. Jena: Friedrich-Schiller-Universität.

von Beyme, Klaus. 1998. Interest Groups in Parliamentary Decisions in the German Bundestag. Government and Opposition 33:38-55.

Binderkrantz, Anne Skorkjær, und Helene Helboe Pedersen. 2017. What is access? A discussion of the definition and measurement of interest group access. European Political Science 16:307-321.

Binderkrantz, Anne Skorkjær, Peter Munk Christiansen, und Helene Helboe Pedersen. 2015. Interest group access to the bureaucracy, parliament and the media. Governance 28(1):95-112.

Bouwen, Pieter. 2004a. The logic of access to the European parliament: business lobbying in the committee on economic and monetary affairs. Journal of Common Market Studies 42(3):473-495.

Bouwen, Pieter. 2004b. Exchanging access goods for access: a comparative study of business lobbying in the European Union institutions. European Journal of Political Research 43:337-369.

Burstein, Paul, und C. Elizabeth Hirsh. 2007. Interest organizations, information, and policy innovation in the U.S. Congress. Sociological Forum 22(2):174-199.

Cianciara, Agnieszka, und Dorota Stasiak. 2019. Der Markt für Politikberatung und Lobbyismus aus der Perspektive polnischer und deutscher Parlamentarier. In Politikberatung und Lobbyismus im parlamentarischen Entscheidungsprozess, Hrsg. Artur Kopka, Dorota Piontek, und Michael Minkenberg, 173-202. Wiesbaden: Springer VS.

Cross, James P., Rainer Eising, Henrik Hermansson, und Florian Spohr. 2021. Business interests, public interests, and experts in parliamentary committees: their impact on legislative change in the German Bundestag. West European Politics 44(2):354-377.

Crouch, Colin. 2008. Postdemokratie. Frankfurt/Main: Suhrkamp.

Culpepper Pepper, D. 2010. Quiet politics and business power: corporate control in Europe and Japan. Cambridge: Cambridge University Press.

De Bruycker, und Iskander. 2017. Politicization and the public interest: when do the elites in brussels address public interests in EU policy debates? European Union Politics 18(4):603-619.

Deutscher Bundestag. 2012. Sachstand: Registrierung und Rechte von Lobbyisten im Parlamentinternationalen Standards. WD 3 - 3000 - 305/12. https://www.bundestag.de/blob/406722/3d2e12695df779ccc 305b65a-89753ba0/wd-3-305-12-pdf-data.pdf. Zugegriffen: 22. März 2020.

Dhungel, Anna-Katharina, und Erik Linhart. 2014. Interessenvermittlung in den Ausschüssen des Deutschen Bundestages. Zeitschrift für Parlamentsfragen 45(4):743-762.

Ebener, Roman. 2020. Augenwischerei statt Transparenz. Blog abgeordnetenwatch.de. https://www. abgeordnetenwatch.de/blog/lobbyismus/augenwischerei-statt-transparenz. Zugegriffen: 19. Okt. 2020.

Eising, Rainer. 2007. The access of business interests to EU institutions: towards elite pluralism? Journal of European Public Policy 14(3):384-403.

Eising, Rainer, und Florian Spohr. 2017. The more, the merrier? Interest groups and legislative change in the public hearings of the German parliamentary committees. German Politics 26(2):314-333.

Flöthe, Linda, und Anne Rasmussen. 2019. Public voices in the heavenly chorus? Group type bias and opinion representation. Journal of European Public Policy 26(6):824-842.

Gerstenhauer, Daniel. 2014. Parlamentarier und Interessenverbände - Lobbying im Bundestag? In Interessengruppen und Parlamente, Hrsg. T. von Winter und J. von Blumenthal, 71-91. Wiesbaden: VS.

Hall, Richard L., und Alan V. Deardorff. 2006. Lobbying as legislative subsidy. American Political Science Review 100(1):69-84.

Hojnacki, Marie, und David C. Kimball. 1998. Organized interests and the decision of whom to lobby in congress. American Political Science Review 92:775-790. 
Ismayr, Wolfgang. 2012. Der Deutsche Bundestag, 3. Aufl., Wiesbaden: Springer VS.

König, Thomas, und Thomas Bräuninger. 2005. Gesetzgebung im Föderalismus. Speyerer Forschungsberichte (FÖV) (237). Speyer: Forschungsinstitut für Öffentliche Verwaltung.

Kriesi, Hanspeter, Anke Tresch, und Margit M. Jochum. 2007. Going public in the European Union: action repertoires of western European collective political actors. Comparative Political Studies 40(1):48-73.

Lindblom, Charles E. 1977. Politics and markets: the world's political-economic systems. New York: Basic.

Marshall, David. 2010. Who to lobby and when: Institutional determinants of interest group strategies in European Parliament committees. European Union Politics 11(4):553-575.

Martin, Lanny V., und Georg Vanberg. 2004. Policing the bargain: coalition government and parliamentary scrutiny. American Journal of Political Science 48(1):13-27.

Marx, Paul, und Peter Starke. 2017. Dualization as destiny? The political economy of the German minimum wage reform. Politics \& Society 45(4):559-584.

Müller-Rommel, Ferdinand. 1988. Interessengruppenvertretung im Deutschen Bundestag. In US-Kongreß und Deutscher Bundestag, Hrsg. Uwe Thaysen, Roger H. Davidson, und Robert G. Livingstone, 300-323. Opladen: Westdeutscher Verlag.

Offe, Claus, und Helmut Wiesenthal. 1980. Two logics of collective action: theoretical notes on social class and organizational form. Political Power and Social Theory 1:67-115.

Olson, Mancur. 1992. Die Logik des kollektiven Handelns. Kollektivgüter und die Theorie der Gruppen, 3. Aufl., Tübingen: Mohr.

Opitz, Stephanie. 2018. Dienstleister für politische Kommunikation in Deutschland. Exploration der Branche durch Typologisierung. Wiesbaden: Springer VS.

Page, Benjamin I., Robert Y. Shapiro, und Glenn R. Dempsey. 1987. What moves public opinion? American Political Science Review 81(1):23-43.

Pedersen, Helene Helboe, Darren Halpin, und Anne Rasmussen. 2015. Who gives evidence to parliamentary committees? A comparative investigation of parliamentary committees and their constituencies. The Journal of Legislative Studies 21(3):408-427.

Pedersen, Helene Helboe, Anne Skorkjær Binderkrantz, und Peter Munk. 2014. Lobbying across arenas: interest group involvement in the legislative process in Denmark. Legislative Studies Quarterly 39(2):199-225.

Rasmussen, Anne, Lars Kai Mäder, und Stefanie Reher. 2018. With a little help from the people? The role of public opinion in advocacy success. Comparative Political Studies 51(2):139-164.

Reyher, Martin. 2015. Eilentscheidung: Bundestag muss Lobbyisten mit Hausausweisen nennen. Blog abgeordnetenwatch.de. https://www.abgeordnetenwatch.de/blog/2015-11-24/eilentscheidung-bundestagmuss-lobbyisten-mit-hausausweisen-nennen. Zugegriffen: 11. Mai 2020.

Sack, Detlef, und Sebastian Fuchs. 2014. Wirtschaftskammern und Parlamente. In Interessengruppen und Parlamente, Hrsg. Thomas von Winter, Julia von Blumenthal, 151-178. Wiesbaden: VS.

Sarcinelli, Ulrich. 2011. Politische Kommunikation in Deutschland. Medien und Politikvermittlung im demokratischen System, 3. Aufl., Wiesbaden: VS.

Schiffers, Maximilian. 2019. NGOs als besondere Akteure der Interessenvermittlung, Zusammenfassende Ergebnisse des Forschungsprojekts „NGOs und Politikmanagement“, Forschungspapier. regierungsforschung.de.. Zugegriffen: 17.10.2019.

Schiffers, Maximilian, und Annika Körner. 2019. NGOs in Prozessen der politischen Interessenvermittlung. Zeitschrift für Politikwissenschaft https://doi.org/10.1007/s41358-019-00203-5.

Siefken, Sven T. 2018. Parlamentarische Kontrolle im Wandel. Theorie und Praxis des Deutschen Bundestages. Wiesbaden: Nomos.

Siefken, Sven T., und Susanne Schüttemeyer. 2013. The German Bundestag and external expertise: policy orientation as counterweight to deparliamentarisation. In Policy analysis in Germany, Hrsg. Sonja Blum, Klaus Schubert, 161-180. Bristol, Chicago: Policy Press.

Speth, Rudolf. 2015. Public Affairs Agenturen. In Lobby Work. Interessenvertretung als Politikgestaltung, Hrsg. Rudolf Speth, Annette Zimmer, 155-172. Wiesbaden: Springer VS.

Spohr, Florian. 2018. Interessen und Informationen in den öffentlichen Anhörungen des Deutschen Bundestages. In Soziologie der Parlamente. Neue Wege der politischen Institutionenforschung, Hrsg. Jenni Brichzin, Damien Krichewsky, Leopold Ringel, und Jan Schank, 309-335. Wiesbaden: Springer VS.

Spohr, Florian. 2019. Hinter verschlossenen Türen, Lobbyisten mit Hausausweis im Bundestag. regierungs forschung.de. Zugegriffen: 31.05.2020. 
Strässer, Christoph, und Frank Meerkamp. 2015. Lobbying im parlamentarischen Bereich - Politiker im Lobbyfokus. In Lobby Work. Interessenvertretung als Politikgestaltung, Hrsg. Rudolf Speth, Annette Zimmer, 219-244. Wiesbaden: Springer VS.

Thomassen, Jacques, und Rudy B. Andeweg. 2004. Beyond collective representation: individual members of parliament and interest representation in the Netherlands. Journal of Legislative Studies 10(4):1-23.

Weßels, Bernhard. 1987. Kommunikationspotentiale zwischen Bundestag und Gesellschaft: Öffentliche Anhörungen, informelle Kontakte und innere Lobby in wirtschafts- und sozialpolitischen Parlamentsausschüssen. Zeitschrift für Parlamentsfragen 18(2):285-309.

Weßels, Bernhard. 2014. Entkoppelung von Parlament und organisierten Interessen? In Interessengruppen und Parlamente, Hrsg. Thomas von Winter, Julia von Blumenthal, 125-148. Wiesbaden: VS.

von Winter, Thomas. 2014. Dimensionen des Korporatismus. Strukturmuster der Verbändebeteiligung in der Gesundheitspolitik. In Interessengruppen und Parlamente, Hrsg. T. von Winter, J. von Blumenthal, 179-209. Wiesbaden: VS.

Wonka, Arndt. 2017. German MPs and interest groups in EU multilevel policy-making: the politics of information exchange. West European Politics 40(5):1004-1102.

Wonka, Arndt, und Sebastian Haunss. 2020. Cooperation in networks: political parties and interest groups in EU policy-making in Germany. European Union Politics 21(1):130-151.

Zimmer, Annette, und Rudolph Speth. 2009. Verbändeforschung. In Politische Soziologie. Ein Studienbuch, Hrsg. Viktoria Kaina, Andrea Römmele, 267-309. Wiesbaden: VS. 